\title{
Assessing Social Concerns Over the Impact of Popular Music and Music Video: A Review of Scholarly Research
}

\author{
D. Atkin ${ }^{*}, 1$ and R. Abelman ${ }^{2}$ \\ ${ }^{1}$ Department of Communication, University of Connecticut, ${ }^{2}$ School of Communication, Cleveland State University, USA
}

\begin{abstract}
For over a half century, the evolution of rock music has been marked by controversy over its social influence. Arguments by the pro- and anti-regulation/censorship camps echo those encountered in debates over the effects of media violence and pornography generally [1]. The present study reviews empirical work on the content and effects of violence in rock music and music videos. In evaluating whether the research meets the high burden for regulatory intervention, we must first establish (1) whether the content of these popular arts is, in fact, providing an increasingly graphic content environment, and (2) whether such contents actually influence audience attitudes and behaviors. A narrative review of the literature suggests that critics of popular music have needed to "fill in the blanks" of their empirical arguments with selective citations to the voluminous literature on general media effects (e.g., with TV violence). The literature on popular music and music videos provides little in the way of longitudinal, externally valid findings that can establish a "smoking gun" with media influences as potent causal agents with human behavior. Implications for media regulation are discussed.
\end{abstract}

\section{INTRODUCTION}

Social influence controversies have marked the diffusion of rock music from the time of its genesis [1]. The gyrations of Elvis Presley in 1956, for instance, prompted Ed Sullivan to call him "unfit for a family audience" [2]. Three decades later, the Attorney General [3] felt compelled to investigate potential antisocial influences of the emerging music television medium. Latter-day critics implicate heavy metal and rap music as causal factors in teen suicide and homicide [4], including a rash of American school shootings in the 1990s that began with a massacre in Columbine, CO [5]. Moreover, as American content continues to dominate international media flows-having been implicated in a recent Finnish copy-cat shooting inspired by Columbineissues concerning popular expression and its influence on children now carry global implications [6].

These debates over rock music are the latest in a predictable raft of social turbulence that accompanies the introduction of new media or art forms [7-9]. To a large degree, arguments offered by the pro- and antiregulation/censorship camps echo those encountered in debates over the effects of media violence and pornography generally $[1,10]$. To what extent do popular music lyrics and music videos produce harmful effects? This question is highly salient now, given the proliferation of new media channels and the dominant position they occupy as public leisure options, particularly among adolescents.

More precisely, the prominence of today's youth culture icons continues to captivate the attention of lawmakers. In a U.S. Congressional Subcommittee hearing focusing on adolescent uses of and responses to this brave new world of media, Senator Joseph Lieberman noted that the new values transmitters are increasingly the gansta rappers, TV

*Address correspondence to this author at the Department of Communication, University of Connecticut, Storrs, CT 06268, USA; Tel: 860-486-3090; Fax: 860-486-5422; E-mail: david.atkin@uconn.edu producers and other players within the media-culture context:

These trend-setters exert an extremely powerful hold on our culture and our children in particular, and they often exhibit little sense of responsibility for the harmful values they are purveying. This music, reinforced by television through MTV and other music channels that present the ganster life as a high life, has spawned its own subculture, setting standards for how to dress, how to treat women and how to resolve conflict cleanly with a bullet [11].

Congress attempted to grapple with this more virulent content environment via the Communications Decency Act (CDA). The Act provided for criminal penalties for anyone who, "by means of a telecommunications device knowingly makes, creates or solicits and initiates the transmission" of content that is "obscene, lewd, lascivious, filthy or indecent" [12]. The Supreme Court in Reno v. the ACLU [13] overturned the CDA, noting that it was overbroad and included forms of speech that may be protected by the First Amendment. Subsequent attempts to ban indecency-which is constitutionally protected in all but the broadcast media-have also been rejected. ${ }^{1}$

Similar issues obtain with nonobscene song lyrics that promote violence; that is, in the absence of a "clear and present" danger to society, attempts to regulate such speech are likely to be found unconstitutional [14]. It's useful, then, to evaluate the nature of scholarly evidence used in support of stricter regulation of popular music expression.

The present essay reviews empirical work on the content and effects of violence in rock music and music videos. When evaluating whether the research meets the high burden for regulatory intervention, we must first establish (1)

${ }^{1}$ A follow-up measure, CCAII, was vacated on similar grounds by a Philadelphia appeals court in 1999 [14]. 
whether the content of these popular arts is, in fact, providing an increasingly graphic content environment, and (2) whether such contents actually influence audience attitudes and behaviors. Since the wide range of content environments and research methodologies auger against a strict meta-analytic approach across domains [15-17] we provide a narrative review of published scholarly research in which these media domains represent a primary focus. Given that the literature uses the terms "rock music" as well as "popular music", we utilize both terminologies as appropriate to match primary source contexts. Our analysis centers on research published since 1965, when rock (and later offshoots like rap) dominated popular music, providing synthesis and criticism for each class of studies.

\section{ROCK MUSIC CONTENT}

Perhaps the most comprehensive review of work on rock music content can be found in Christensen and Robert's [16] compendium, It's not only rock and roll, which provides a good starting point for review (and critique) of empirical evidence, one that can help determine whether the high burden for government regulation [18] has been met. The main points of that volume were aptly summarized in Robert's [19] testimony before Congress:

* For the most part we are talking about two genres of music--heavy metal and rap...popular music has fragmented into a wide array of sub-genres. Billboard magazine now charts over 20 different types of music, and the Grammy Awards currently recognize 80 different categories, many of which represent distinct music genres. This is important because, by middle adolescence, kids begin to gravitate toward one or two of those many categories;

* Different studies estimate that such content occurs in anywhere from $25 \%$ to $70 \%$ of metal and rap songs and/or videos depending on the definitions (or sex and violence) used;

* The lyrics are quite explicit and concrete--and seem to have become more so over the past five or six years;

Christenson and Roberts [16] conclude that popular music deals with central adolescent issues, including the following themes:

The consequences of behavior--even antisocial behavior--in pop music are often positive. Sex is usually safe, rudeness is cool, threats toward police or other authorities are rarely punished, and tatoos are never for life (p. 222).

Although several content analyses of rock music were conducted during the 1960s and 1970s [20] Christensen and Roberts' [16, p. 120] review concludes "there are no published analyses of lyrics in the 1980s and early 1990s careful and thorough enough to provide a solid basis for gauging current trends". In updating and extending their framework beyond 1997, the present analysis confirms that there remains a dearth of work in this area [15].

Earlier work [20, 21] found that, by the early 1980s, only about $50 \%$ of the songs on the Billboard charts dealt unambiguously with romance, sex, or love. Albert [22] found that intensity and violence represent the most important factors distinguishing rock songs, while evaluations of beauty, interest and goodness were relatively less important.

A review of unpublished data from Roberts [cited in 16]), addressing the primary themes in the Top 40 hits for each even-numbered year (1980-1990), identified the following central adolescent issues: love and relationships (70\%), dance and music themes (7\%), social and political issues $(5 \%)$, identity and growing up (5\%), and loneliness (3\%); another $8 \%$ covered a range of miscellaneous topics.

The authors [16] conclude that the audience's orientation toward "deviant or unusual messages" invites an adversarial stance among scholars, as the:

...well documented tendency of some music lyrics and videos to celebrate deviant and nonnormative behavior (e.g., premarital sex, drug use, violence and suicide), most public attention to the content-related effects of popular music has reflected a concern with negative effects...the preponderance of empirical work reflects the same bias.

Placing this work in historical context, Allen et al. [15] note that "(j)azz, ragtime, swing, rock and roll, punk, rap and grunge are examples of types of music that appealed to the young and offended the more conservative or older members of the society." Industry defenders [2] argue that, because the use of such terms as "deviant" and "nonnormative" are value-laden descriptors of song lyrics, researchers should not take original research findings at face value.

\section{Critique and Summary}

When acknowledging critical biases in the literature, scholars point out the anecdotal nature typifying most critiques of the music industry. Christenson and Robert's research corpus, which dominates the scholarly record before Congress on the matter, concedes [16] that "[W]e do not know in specific terms what proportion of songs incorporate violence, although it is probably not a great proportion in the very top songs." This conclusion is based on work [23] indicating that only $8 \%$ of the songs in a 1980-1990 top 40 analysis contained references to violence, and violence or death formed the primary focus in fewer than $1 \%$. This may be a conservative estimate, given that the criterion time frame largely omits the onset of rap. Yet, ironically, this literature offers similar generalizations to support the assertion that lyrics have grown more explicit in recent years.

Thus, the acknowledged dearth of research doesn't preclude commentators, particularly in the popular press, from drawing harsh conclusions about the impact of popular music [5]. The problem with this type of evidence involves the opportunistic way in which industry assailants might select the data to bolster their criticism. As Christenson and Roberts [16] themselves note, a single case study could advance the improbable conclusion that contemporary music (e.g., "Hanson Live") is less sexually than before, based on a comparison involving the following example from the 1920s:

He's a deep deep diver, with a stroke that can't go wrong Oh, he can touch the bottom, and his 
wind olds out so long (citing lyrics from Bessie Smith's "Empty Bed Blues").

As they and others conclude, this potential for the (ab)use of selective examples to document musical trends--perceived or imagined--underscores the need for carefully constructed, large and representative quantitative analyses of music content. The need for such rigor becomes manifest in the larger public policy debate, given the important First Amendment and public safety issues at stake here.

The absence of any timely work addressing violence and music, however, leaves industry critics groping for evidence in support of regulation. Of course, after the introduction of MTV in 1981, much of the research effort that might have been devoted to music lyrics perhaps redirected toward the emerging video art form, which is reviewed in turn.

\section{MUSIC VIDEO CONTENT}

Over the past two decades, critics have decried the level of violence and misogyny portrayed in music video [24]. Feminists [25] maintain that videos objectify and often brutalize women, promoting acceptance of rape myths and ultimately paving the way for real-life violence against women. As NCTV head Thomas Radecki (quoted in [26]) notes, "[T]he message is that violence is normal and $\mathrm{OK}$, that hostile sexual relations between men and women are common and acceptable, the heroes actively engage in torture and murder of others for fun." An early NCTV study itself, addressing 160 hours of music videos, estimated that viewers are exposed to an average of 18 instances of violence per hour (or nearly one every four minutes) [27].

At about that same time, Sherman and Dominick [28] found that over half $(57 \%)$ of concept videos appearing on various channels contain violent acts $(m=2.9$ acts per concept video). Their standard was a bit more restrictive than that of the NCTV study, focusing on "overt" expressions of violence (as opposed to threats). Violent episodes occurred in $56.6 \%$ of the sample, with males--who outnumber females 2-to-1 overall--accounting for about $75 \%$ of the aggressors and victims. Hand-to-hand combat was the most common form of aggression, and males were more likely to be injured than females. The violence shown typically did not portray any consequences of aggression. Only $3 \%$ of the violent acts resulted in death, while $12 \%$ resulted in injury.

Baxter et al. [29] found a comparable (53\%) proportion of videos containing any violent acts or crime. Their analysis was based on a sample of 62 MTV videos utilizing 23 content categories. They noted, in particular, that $25 \%$ of the videos showed violence against people, while a sixth featured dance movements that mimicked violence. Only 2 of the 62 videos showed homicide. Some $10 \%$ of videos showed guns, knives or other dangerous weapons and-consistent with Sherman and Dominick [28]--most of the fighting portrayed was hand-to-hand. Similarly, Davis [30] discovered that $44 \%$ of concept videos contained nihilistic images.

Even so, other early investigations found some unusually counter-patriarchal features with music videos. Sherman and Dominick [28] note that women are more likely than men to initiate aggressive acts, perhaps representing an expression of backlash against strong women by an overwhelmingly
$(90 \%+)$ male production corps. The authors termed this reversal of sex roles the "predatory female" stereotype of music videos. With regard to other socio-demographics, Brown and Campbell [31] found that blacks are more likely than whites to engage in pro-social acts and sexual acts, although less likely to behave antisocially.

Mcdonald and Estep [32] conducted an examination of crime portrayed on prime-time TV and MTV during the 1987-88 and 1988-89 seasons. They were investigating the claim that MTV is more violent than the major commercial networks. Study results indicate that criminal violence on MTV dropped from earlier levels. The authors noted that violence in both forms of TV could be separated into three distinct categories: (1) humorous depictions (e.g., comedy and satire); (2) realistic portrayals (e.g., with war or social protests), and gratuitous violence, or that which is used for dramatic purposes.

In one of the most comprehensive early investigations Kalis \& Neuendorf [26], note that the new music video medium allowed popular music to reach a large audience visually as well as aurally. Their own study of 14 hours of MTV content during 1985 focused on: "(1) the occurrence of and audience validation of aggressive cues, (2) the prominence and cue type, and the initiators and recipients of, validated aggressive cues, and (3) the pacing, videotape and real time length of music videos" [26, p. 153]. The authors found that violence was more pervasive than was found in prior investigations, a difference that may be attributable to their late 1980s time frame. Even so, they [26, p. 153] note that "aggressive cues may be less prominent in music videos than common criticisms would lead one to believe." In particular, $13 \%$ of all shots contained aggressive cues, constituting $9 \%$ of all video time, and nearly $40 \%$ of the videos had no validated aggressive cues; concept videos contained more aggressive content than did performance videos, but the overall portion was still minor, less than that available on prime-time network TV.

With regard to gender, women were less than a third as likely as men to be the objects of violence, in quantitative terms. But violence against women was shown in a distinctive way, qualitatively, with the camera more likely to linger on them, particularly with close-ups or extreme close-ups.

Extending this work through the early 1990s, Tapper et al. [33] investigated the differences between types of music videos, as classified by their musical genre. The study was based on an analysis of eight half-hour segments recorded across four days from Black Entertainment Television, The Nashville Network, MTV and VH-1 $(\mathrm{n}=$ 168 hours for analysis). They found that the visual elements vary quite widely, to the point where it's wrong to consider music videos as homogeneous. Contrary to studies conducted during the 1980 s, they found that violence occurs rarely and is not a function of musical genre.

Jones [34] notes that newer genres of music videos, including rap and hip-hop, have intensified the debate on the negative effects of this music form on its audience. His study of 203 videos from popular video channels investigated the occurrence of elements of sex and violence based on five music styles: rap, hip-hop, rhythm and blues-soul, country and western and pop. Study results reveal that none of these 
genres predicts elements of the occurrence of more physical aspects of sex and violence. Even so, rap videos were consistently higher than other genres in certain other behaviors (e.g., gun talk, drug talk, gambling, and the presence of alcohol). This study, although now somewhat dated, is among the most up-to-date in the literature; it thus escapes the flaws that characterize other studies, many of which reflect decade-old music, and thus fail to reflect the growth of rap, etc.

More recently, an analysis of 1998 programming was touted as the most extensive ever conducted on violent content in entertainment [35]. Prepared by the nonpartisan Center for Media and Public Affairs, the study found that there was an average of one act of serious brutality for each four minutes of programming in the visual media. This rate of incidence did not vary much by modality, as the study analyzed portrayals shown in film, entertainment television programs and music videos. The study authors further commented that violence was often portrayed as harmless or without consequence:

"It was used by heroes nearly as often as villains" [35, p. 1].

Much of the research focus on media violence has shifted to emerging channels such as videogames and the Internet $[6,36,37]$, although scholars have yet to fully examine content delivered through those modalities. While subsequent work has examined changing music lyrics in the context of effects [38], the relative decline in contentanalysis work on music video since the mid 1990s stands testament to the rapidly changing media environment with which adolescents (and scholars) are now confronted. We'll move on to explore those influences in subsequent sections. Even in today's rapidly changing information grid, however, popular music remains a staple accounting for 1.5 to 2.5 hours of an average American adolescent's media diet [39].

\section{Critique and Summary}

Aside from the time lag inherent in published research, most of the content analyses of music video employ significant flaws. First, most use a "one shot" design, failing to provide a longitudinal perspective across several years to truly identify patterns in content. Comparing a collection of studies performed by different scholars using different methodologies, often implemented during different years, fails to provide an accurate account of content over time. Yet, many pro- and anti-regulation groups do just that and generate their recommendations accordingly. Second, the number of data points or subjects included in this research is typically low. Such selective sampling likely reflects logistical constraints characteristic of academic research, where person-power and budget limitations restrict the breadth and scope of a given scholarly enterprise. This, in turn, limits the generalizability of study conclusions. In addition, the highly diverse nature of the content under study does not readily lend itself to simple categorization in a "convenience summary", such as that provided for song titles in Billboard magazine.

These limitations and inconsistencies serve to explain, at least in part, the remarkable variation in findings across studies identified here. Even during comparable periods, violence estimates range form $25 \%$ of all videos [29] to roughly $60 \%$ of videos [28]. Findings concerning the nature of the violence in popular videos are equally inconsistent across studies. Even when time or content confounds are minimal, differences in coding schemes threaten the reliability of the literature base, alongside the generalizability of reported findings. The significance of these choices that researchers must make, particularly when they're establishing a "representative" baseline for music video content, is even more pertinent in the realm of media effects studies reviewed in the section to follow.

\section{ROCK MUSIC USES AND EFFECTS}

Allen et al. [15, p. 263] note that, "(f)or each generation, a new iteration of popular music creates new allegations of antisocial effects". As with studies of rock music content, the subject of music listenership attracts relatively little research interest, in comparison with music television. Early work in this area examined personality-oriented themes and listening patterns in teen-age music and its relation to selected peer and academic variables beyond the scope of our analysis [40].

More recently, Lewis [41] explored the influence of popular music on drug use among youth. His large scale survey of 2,950 high school students uncovered a positive relationship $(\mathrm{r}=0.275)$ between listenership and drug use tendencies. Similarly, when contrasting uses of television and music in adolescent life, Larson and Kubey [42] surveyed students around the age of 16 . They found a positive correlation $(\mathrm{r}=0.31)$ between use of music and selected antisocial tendencies. However, Tanner's [43] survey of 452 Canadian high school students failed to find an appreciable link between exposure to music and respondent attitudes.

Prinsky and Rosenbaum [44] conducted a survey comparing adults' impressions of rock music with those of teenagers. Among the "vast differences" found between these groups, the authors note that youths reported hearing subjects relating to their lives, such as "growing up". By contrast, adults heard more references to sex and violence. The authors suggested that the differing impressions between adults and teens reflect differences in experience, learning, and literary abilities. Although this study demonstrates that individuals naturally prefer stimuli that reflect their values and attitudes, it reflects a limitation common to research in this tradition: the results observed may be due to factors other than the criterion music under study.

Wanamaker and Reznikoff [45] conducted an experiment in which participants responded to recordings of songs. They found no differences in college students' levels of hostility in response to "aggressive rock music." Respondents $(\mathrm{n}=10)$ were broken down into identical groups who wrote stories about each of five ambiguous pictures while listening to a recording of (1) aggressive music and aggressive lyrics, (2) aggressive music and non-aggressive lyrics, or (3) nonaggressive music and nonaggressive lyrics. After the songs were repeated continuously for 20 minutes while the students wrote their stories, the Buss-Durkee hostility scale was administered. Contrary to expectations, no differences were detected among the three groups in the amount of 
hostility expressed in the stories (nor in scores on the hostility scale). The authors uncovered some evidence that students did not attend to or understand the lyrics in the songs, suggesting that effects cannot manifest themselves unless differences in content are apparent. These results are intriguing, as experiments typically generate higher-level correlations (although they're often criticized for their artificiality and lack of generalizability).

Proceeding on that point, St. Lawrence and Joyner [46] conducted another audio-only study on the effect of listening to heavy metal music. The authors investigated the influence of listening to sexually violent heavy metal on acceptance of sexually violent behavior and gender-role stereotypes. Seventy five male students at a southern university were split into groups that heard either: (1) sexually violent heavy metal rock, (2) Christian heavy metal rock, or (3) easy listening classical music.

Student orientations were tapped with a questionnaire mailed a month before and immediately after the listening session. The instrument addressed adversarial sexual beliefs, gender-role stereotyping, acceptance of rape myths (e.g., women enjoy it) and sexual arousal frequency; the preexposure instrument also included covariate measures on religious orientation.

Contrary to expectations, results suggest that musical content matters less than form, as influences did not vary between the violent heavy metal and Christian heavy metal groups. Both formats produced more negative attitudes toward women than the classical music stimulus; lyrics, then, were less important than the musical genre in which they were found. Also, subjects with extrinsic religious orientation indicated higher agreement with sexist and rapesupportive beliefs than their intrinsically oriented counterparts. Interestingly, classical music produced the greatest level of sexual arousal.

As with the Wanamaker study, these findings leave open the issue of whether students really heard or understood the lyrics presented to them. St. Lawrence and Joyner [46] suggest that their subjects were able to correctly identify the base genres of musical stimuli (classical, rock, etc.), so a minimal baseline of attention can be established. One limitation involves the fact that there was no measure of lyrical understanding, aside from sorting the music into the categories mentioned above (e.g., labeling sexually explicit music as Rock). Thus, students may have merely been indicating previously learned associations of sound (heavy metal) with "sex and violence", rather than responding to specific lyrics.

Arnett [47, 48] studied the uses and effects of heavy metal music on adolescents, using a purposive survey of 245 17 year-old students. He was particularly interested in relationships between heavy metal listening and reckless behavior among adolescents. Consistent with other work in the area, Arnett found a modest positive relationship $(\mathrm{r}=$ 0.188 ) between heavy metal listening and antisocial behavior. A concurrent study by Bleich et al. [49] examined enjoyment of defiant rock music as a function of adolescent rebelliousness. Using an experimental design $(n=84$ students), the authors discovered a relatively strong $(\mathrm{r}=$
0.384) relationship between consumption of rock music and antisocial behavior.

More recently, Johnson et al. [50] assessed the effects of exposure to nonviolent rap music on perceptions of teen dating violence. Their experimental subjects were 30 male and 30 female African American adolescents (aged 11-26 years). These youths, drawn from an inner-city youth club in Wilmington, NC), were exposed to nonviolent rap videos containing images of women in sexually subordinate roles. There were compared to a group who saw no videos (the "no exposure control" group); this group then read a vignette featuring teen dating violence initiated by a male. The authors found a significant interaction between gender and video exposure. In particular, acceptance of the use of violence did not vary in relation to exposure for male subjects.

Yet female subjects exposed to the videos displayed a greater acceptance of the violence than females who were not exposed. It was crucial that participants believed that they were involved in two different, unrelated experiments (which, in reality, was not the case). In that regard, the study's intention was well disguised from the participants, making this one of the better-designed criterion studies.

At about the same time, Smith [51] investigated the effects of exposure to violent lyrics in heavy metal music--in conjunction with a low dose of alcohol--on aggressiveness. Participants, college-age males, were allowed to aggress against a fictitious confederate in a modification of the Buss aggression paradigm via an aggression machine. Results indicate that participants exposed to highly violent lyrics delivered shocks of longer duration to the fictitious confederate, compared to those exposed to less violent lyrics. In addition, participants administered shocks of increasing intensity and duration across trials. The authors note that these results are similar to those found in studies on the effects of visual depictions of violence in television and film media, in that aggression effects may also be affected by non-visual media.

Barongan et al. [52] investigated the influence of misogynous rap music on sexual aggression against women. This experiment was designed to isolate the effects of cognitive distortion addressing females on sexually deviant or aggressive behavior. Some 54 subjects were drawn from the male undergraduate population at a midwestern university. Subjects were split into two groups, one of which listened to misogynous rap music, while the other listened to neutral rap music. Participants next viewed neutral, sexualviolent, and assaultive film vignettes, selecting one to show to a female confederate.

From within the subjects in the misogynous music conditions, some $70 \%$ showed the neutral vignette and $30 \%$ selected the assaultive vignette. Among the subjects in the neutral condition, only $7 \%$ showed the sexual-violent or assaultive vignette, while $93 \%$ showed the neutral vignette. Relative to those showing the neutral vignette, subjects showing the sexual-violent or assaultive vignette noted that the female confederate was more upset and uncomfortable. Findings support the relationship between cognitive distortions and sexual aggression, as misogynous music facilitates sexually aggressive behavior in males. In addition 
to the generalizability concerns accompanying other studies, the present experiment could be criticized for utilizing a relatively tangential (and homogeneous) audience segment: highly educated white males.

Similarly, Dixon and Linz [18] examined the impact of rap music on sex, attitudes and beliefs. Utilizing an experimental design $(n=172$ college students), the authors discovered a significant positive correlation $(r=0.3)$ between exposure to rap and antisocial attitudes.

Concurrent work by Webster et al. [53] explored the influence of sexually violent rap music on attitudes of men with little prior exposure. In particular, the study focused on commercially available, sexually violent rap music (i.e., "gangsta" rap) and its influence on attitudes toward women. Some 60 male undergraduates were drawn from a small midwestern university with a religious affiliation. These subjects were exposed to (1) music, (2) lyrics, (3) both music and lyrics and (4) neither. In this way, the authors were able to isolate the effect of gangsta rap music and lyrics from each other (and disentangle the effects of acclimation to gangsta rap). Considering aggregate numbers across all attitude measures, more powerful (negative) attitudes toward women were generated by music-only and no-treatment groups than lyrics alone or with music. The lyrics condition generated significantly higher levels of agreement with adversarial sexual belief items than the no-lyrics condition.

Shifting to direct behavioral influences of heavy metal, Christianson and Roberts [16] recount a raft of anecdotal reports implicating heavy metal in teen suicide:

Heavy metal attracts a coterie of highly involved, highly absorbed fans, primarily (but not exclusively) White males in the middle adolescent years. These youth are more likely than others to be at odds with parents, school, and "authority", and tend to be risk takers...a significant subgroup of fans who are troubled or "at risk".

Focusing on nihilistic adolescents, heavy metal music and its influence on paranormal beliefs, Trostle [54] surveyed 66 high school aged individuals. He uncovered a relatively strong $(\mathrm{r}=0.763)$ correlation between exposure to heavy metal music and paranormal beliefs.

Martin et al. [55] investigated the influence of heavy metal on suicide by in a study of 200 Australian high school students. They found that (1) heavy metal fans strongly manifested several "troubled youth" attributes, and (2) a preference for heavy metal music was directly associated with suicidal thoughts and behaviors. In particular, over $20 \%$ of males and $60 \%$ of females in the metal fan group indicated that they tried to harm or kill themselves during the preceding six months; these figures were closer to $8 \%$ of males and $14 \%$ of females among the pop listener group.

Similarly, Stack et al. [56] examined the suicide issue, using a different approach to establish whether teenage subscribers to heavy metal magazines were "likely to be immersed and committed to the symbols and messages communicated through the subculture" [56]. In the absence of any national measures of heavy metal exposure, the authors assumed that a baseline could be drawn from Audit
Bureau of Circulation data on teenage subscriptions to Metal Edge. The information was adjusted for the size of the 15- to 24-year-old population and used for each of the 50 states in 1988.

Study results indicate that a strong heavy metal subculture in a given state was strongly related to a higher suicide rate. This link between suicide and heavy metal subscriptions survived even when mediating factors like race and divorce were taken into consideration. The authors note that this finding is consistent with earlier work on adult suicide rates in relation to listenership of country music [57].

At about the same time, Ballard and Coates [58] conducted an investigation of the impact of lyrical content (or music type) on the moods of 164 male undergraduates (m age $=19.7$ years). Subjects heard one of the 6 songs where two music types (heavy metal and rap) were crossed with three lyrical content themes (nonviolent/control, homicidal, or suicidal lyrics), and asked to complete a memory task. The subjects completed different mood inventories to assess anger experience and expression, levels of depression, selfesteem, mood, state- and trait-anxiety, and current suicidal ideation.

The authors found no significant effects of lyrical content or music type on suicidal ideation, anxiety, or self-esteem. Higher depression scores were noted for nonviolent rap songs than violent rap songs. Higher levels of angry responses were noted for rap songs than for heavy metal songs. The authors concluded that there is no evidence that lyrical content or music type has an immediate effect on either state anxiety or suicidal ideation.

Brown et al. [59] assessed whether exposure to sexual content in music, movies, television and magazines in early adolescence predicted sexual behavior in middle adolescence. Overcoming the limitations of one-shot studies in this area, the authors examined an in-home longitudinal survey of 1017 black and white adolescents from 14 middle schools in North Carolina. Respondents were interviewed initially at age 12 to 14 years of age, and again 2 years later, via a computer-assistant self interview method. Study results suggested that exposure to sexual content through these various modalities accelerates white adolescent sexual activity and increases their propensity to engage in early sexual intercourse. Black teens, by contrast, were more powerfully swayed by their friend's sexual behavior and parent's expectations than by exposure to media content.

Martino et al. [39] conducted a national longitudinal telephone survey of 1461 adolescents to test longitudinal associations between exposure to music lyrics and later changes in sexual experience (e.g., intercourse initiation). Initial interviews were conducted when respondents were 12 to 17 years old (T1), and again 1 and 3 years later (T2 and T3, respectively). Study results suggested that those listening to more degrading sexual content at T2 showed a greater likelihood of initiating sexual intercourse and other sexual activities. However, listening to music with non-degrading sexual content bore no such relationship with respondent sexual behavior. The authors concluded that reducing adolescent exposure to degrading sexual content in popular music could delay the initiation of sexual behavior. 
Finally, Allen et al.'s [15] meta-analysis of 11 studies found a modest positive relationship (average $r=0.186$ ) between exposure to music and selected antisocial behaviors. The authors noted some divergence in results between the nonexperimental and experimental data sets examined, with the latter generating larger observed effects. On balance, the magnitude of these effects is not inconsistent with the relatively weak media effects found in domains ranging from music videogame violence to advertising [17].

\section{Critique and Summary}

Experimental studies on music effects could of course be criticized for their artificiality, given that a true experimental manipulation of suicide influences would be unethical. But the studies of Stacks and his associates--based on national data--succeed more than most in overcoming limitations in external validity (generalizability) that characterize most experimental or school-administered surveys. That said, large scale purposive samples of the sort employed by Brown et al. [59] do much to enhance the external validity of that method, while adding an important longitudinal dimension that strengthens attributions of causality.

By the same token, experimental studies are distinguished for their stronger internal validity; that is, they are able to more directly measure what it is they purport to measure, relative to surveys, and thus can generate more powerful effects in the form of stronger correlations between exposure and effect. But that strength can be a weakness when scholars try to project of laboratory findings in the real world. In short, not many college students listen to music in the way that college samples--even assuming that they're representative of the population--are asked to do in a laboratory setting. Moreover, few these studies endeavor to provide a compelling disguise for their enterprise, thus sensitizing subjects to their potential purpose.

In addition, experimental stimuli are typically selected to elicit the greatest response contrasts, including especially compelling lyrics about, say violence against women (for a treatment group). This might be juxtaposed against remarkably tame footage to be shown as representative of a control stimulus. Although useful in generating large contrasts suitable for scientific examination, these false polarities generally fail to replicate the ways in which audiences attend to music at home, where listening is typically a secondary activity. Moreover, in a more naturalistic setting, audiences are distracted by coviewers, interruptions, and the like. Their understanding of lyrics-found to be low in a number of studies [48]--is also likely to be lower when listening outside of a controlled lab setting.

These various and several mediating factors would likely serve to weaken the effects of a media stimulus in real-life, so it is difficult to truly know what--if anything--these lab experiments are really measuring. Many of those addressing general music video effects are reviewed in turn.

\section{MUSIC VIDEO USES AND EFFECTS}

Although research on music video effects also represents a challenging domain, in terms of disentangling causal agents, the stories told by popular videos should be easier to decipher than those accompanying the provocative tones of heavy metal. Perhaps, for that reason, the strength of evidence in support of media influences has generally been more compelling than that indicated for audio recordings.

Focusing on exposure dynamics, Sun and Lull [60] surveyed a sample of 452 respondents on their uses and use motivations concerning music video. They noted a weak $(r=$ 0.195 ) relationship between exposure and selected attitudinal measures, although antisocial behaviors were not a primary focus of their study.

Greerson and Williams [61] conducted a study of seventh and tenth graders from working class homes enrolled in a small-town school in southwestern Ohio. They investigated the impact of MTV segments, selected randomly for high impact thematic content and shown to half of the subjects within each grade. The other half were shown a "highimpact" stimulus featuring heavy doses of sex, violence and anti-establishment content. In the experimental group, subjects were shown 30 minutes of MTV segments and then given a survey addressing adolescent attitudes toward parental influence, premarital sex, violence, drug use and MTV itself.

Results indicate that (1) the views of tenth graders more closely approximated the views of their parents than did those of the seventh graders; (2) significantly more adolescents agreed that premarital sex was acceptable after viewing MTV (for both high-impact and random video groups), and (3) there was no connection found between MTV viewing and drug use or violence; the high-impact video group did express lower levels of disapproval of violence, however. Although this and other experimental designs, could be criticized on the basis of artificiality, it provides a fairly realistic, ethically-sound treatment of a difficult-to-research subject.

Walker [62] examined the relationships between MTV viewing and exposure to other sources high in violent content in TV, motion pictures and books. These media were measured with a survey of 116 seventh graders and 107 high school juniors in greater Waterloo, Iowa during 1982 and 1983. The subjects recorded their daily exposure to television for one week and completed a questionnaire to measure exposure to books and film during the previous two months (categorized as high or mild violence types). By definition, MTV was categorized as a high violence type. Results indicate that, for eleventh graders, MTV viewing is inversely related to exposure to other sources of violent content (and positively related to viewership of mild violence daytime soaps and mild violence books). In this fashion, exposure to MTV was not significantly related to perceived violence in society. The author thus concluded that concern over the levels of violence in videos may be exaggerated.

Peterson and Phost [63] investigated the influence of music video on attitudes towards violence against women. Their experiment involved 144 undergraduate males who were shown one of four schemes of music video content: (1) erotic-nonviolent, (2) erotic-violent, (3) nonerotic-violent, and (4) nonerotic-nonviolent. Upon the completion of their viewing, subjects filled out a questionnaire, the "Student Sexual Attitudes Scale", addressing acceptance of rape myths, adversarial sexual beliefs and the like. Other 
instruments addressing student mood states and sexual arousal were then administered. The only group of the four above to register a significant effect involved non-erotic violent images. No significant differences were observed among the other three groups. The authors concluded that the erotic-violent videos may have failed to elicit a negative response because they were countered by pleasurable erotic content. However, given that the intent of this study was not fully disguised, it's difficult to draw firm conclusions from the study. The authors [63, p. 327] nevertheless cautioned that some videos prompt a "calloused and antagonistic orientation toward women."

Hanson and Hanson [64] examined the influence of antisocial content in rock music videos. They utilized a twopart experimental design to see if these videos would increase acceptance of antisocial behavior. The study was well-disguised as a job interview situation where (college student) subjects were told they were to evaluate candidates. While waiting for their "interview", subjects were asked to view either three antisocial or three neutral videos.

Subjects were then exposed to (or "accidentally observed") a staged event in which, after telling a joke, one applicant was bluntly warned to "settle down" by an authority figure. As the figure was leaving the room, half of the subjects saw the confederate "job applicant" make an obscene gesture towards him. The remaining half saw the confederate merely adjust his clothing after receiving the rebuke. All told, this scenario generated four treatment groups: (1) antisocial video/obscene gesture, (2) antisocial video/no gesture, (3) neutral video/obscene gesture, and (4) neutral video/no gesture. Subjects were then shown a taped interview of the two applicants and administered a questionnaire tapping their feelings towards each.

Study results suggest that those who saw the neutral videos liked the job applicant less, as expected, and evaluated him less favorably if they saw the obscene gesture. By comparison, those who watched the antisocial videos did not like the applicant any less when he made the obscene gesture, suggesting that a relatively brief exposure to antisocial videos nullified any disdain one might feel towards a rude person.

Utilizing a similar pool of student participants, Hanson and Hanson [65] examined the influence of sex and violence on the appeal of rock music videos. In particular, they designed two experiments to assess emotional responses to sex and violence. Again, utilizing an elaborate cover story, students were shown varying levels of sexual content. Subjects in Experiment One demonstrated positive emotional responses to sexual content. Results from Experiment Two indicate that viewers did not enjoy violent videos and experienced negative responses to violent videos.

Miller [66] conducted a study on the effects of music videos on adolescent meaning construction and attitudes toward physical violence as a method of conflict resolution. Where past work suggests that viewing violence in the home and on television is a predictor of aggression in individuals, this study investigated the influence of music videos that present conflict resolutions in domestic violence situations. Some 106 adolescents responded to a survey on the portrayal of males and females in music video. They were then shown three current videos featuring the following themes: (1) an abused female responding aggressively, (2) an abused female acting in a prosocial fashion, and (3) an abused female responding regressively. Measures assessed the effects this conflict resolution mode had on adolescent participants and the degree to which they comprehend themes in the videos themselves. The author found that, consistent with expectations, subjects who watched the most violent video selected the most aggressive responses. Female respondents were less retaliatory than their male counterparts, while subjects exposed to the regressive video were more despondent. Findings indicate that moods, cognition and attitudes can be affected by even a short exposure to music video.

Focusing on the reduction of aggressive behavior after removal of music television, Waite et al. [67] conducted an experiment with 52 subjects aged in their late 20s. They found a moderate positive correlation $(r=0.327)$ between exposure and measures of antisocial behavior.

Walbott [68] examined the effects of sexual and aggressive content in video clips on student viewers. Subjects included 40 students drawn from a German university (with a mean age of 25 years). They were randomly assigned to one of two groups that viewed either (1) the audio component of 30 music videos, or (2) both the audio and video component of those videos. Each video contained equal parts of neutral, sexual or aggressive content, to which subjects were asked to record their impressions of emotions conveyed. Results suggest that these responses can be understood in relation to the content and medium, reinforcing past work suggesting that subjects attend more readily to combined audio-video stimuli than audio-only modes.

Zillmann et al. [69] investigated the impact of radical rap music video on ethnic divisions and academic self-esteem. In the first portion of the experiment, high school students were shown a set of music videos from among the following: (1) four popular rock videos, (2) four nonpolitical rap videos, or (3) four radical political rap videos. When done viewing, students responded to items tapping evaluations of the video and self-esteem. Students then heard speeches by one of six apparent candidates in a "second study" that was a welldisguised extension of the rap treatment. In this phase, students heard the candidates--three of whom were White and three of whom were Black--advocate one of three different political platforms: (1) racially radical, (2) neutral, or (3) liberal. After hearing each speech, the students were asked to evaluate the candidate and answer items about their own academic-esteem.

Results suggest that the music video conditions had no effect on political evaluations and self-esteem among Black students. Exposure to popular music videos did negatively influence scores on the self-esteem subscale among white students. In particular, exposure to radical rap strongly impacted the views and candidate selections of white teens, making them more tolerant (as opposed to the expected ethnically defensive response).

A similar two-panel study by Johnson et al. [70] assessed the effects of exposure to rap music on the attitudes and perceptions of African American Males (aged 11-26 years). 
The violent videos featured images of weapons and violent acts along with lyrics that condoned violence (e.g., "Roughneck" by M.C. Lyte; "Hazy Shade of Criminal" by Public Enemy). Some 46 subjects were exposed to music videos featuring violent rap, nonviolent rap, or no music videos (a control group). Subjects were read 2 "vignettes" involving (1) an incident incorporating themes of violence and (2) a situation involving a young man who had academic aspirations and a friend who chose a dubious route to materialistic gain.

Results suggest that subjects in the violent rap condition expressed greater acceptance of the use of violence and reported a higher probability that they would engage in violence, in comparison with the subjects in the nonviolent rap conditions. Moreover, compared with the control group, subjects in both rap video exposure conditions were more likely to say that they wanted to be like the materialistic young man. They also expressed less confidence that the other young man would achieve his educational goals.

In the most recent study of music video influences, Peterson et al. [38] investigated the impact of sexual stereotypes in rap music videos on African American adolescent females (survey $n=522$ ). They found that adolescents perceiving more such portrayals were more likely to test positive for marijuana, engage in binge drinking, have a negative body image, and have multiple sexual partners.

\section{Critique and Summary}

The above studies share limitations common to humansubjects research, given the limitations in study design, lack of a "true" control group (i.e., comparison person that has never been exposed to such content), human subjects treatment constraints, etc. As Roberts [19] concluded in his testimony before Congress,

This often leads critics to claim the research is irrelevant; they would, it seems, have us expose kids to violent videos, then count how many women they attacked, fights they instigated, or shots they fired. ...when working with issues such as sex and violence, ethical considerations make behavioral outcomes extremely difficult to study.

We concur that such a burden is extremely difficult to meet, but is not altogether unattainable; the literature, in particular, offers promising examples. However, as outlined in the preceding critique of work on the effects of recorded music, the validity of work in this area is limited by a heavy reliance on experimental as opposed to more externally valid cross-sectional methods.

It's important to further acknowledge the problems in generalizing about the social impact of popular music from, say, a college student sample to the rest of the population. The same is true when commentators take findings from studies performed in the 1980s and generalize their results to today's audience. Today's young adults share little in common with previous generations, particularly in terms of their media and recreation habits. Abelman [71] profiles the distinctive qualities, characteristics and media habits of postBaby Boom teens:
Generation $\mathrm{X}$ is comprised of highly critical, extremely media-literate consumers who take from the media what they need and what they find entertaining, but do not accept information from the media at face value...They always seek to control the communication at their disposal. The media habits of Generation $\mathrm{X}$ are as diverse as the people within it. To suggest that one can generalize from a sample of today's students to the general population-particularly regarding issues of media use, gratifications and impact-is sheer folly.

Focusing on the positive attributes of this research tradition, work on music video influences is more likely to emulate real-world media consumption practices than that addressing music listening. Where the latter may be influenced when an experimenter specifically draws a subject's attention to lyrics--which they otherwise might not notice listening at home--the meanings inferred from music video carry a greater potential for clarity (especially when TV viewing is a primary activity). Even so, results garnered from music video studies, even those that are well-disguised, may tend to overstate the effects of a given stimulus. This "high correlation" dynamic again reflects the fact that subjects are typically shown widely varying stimuli, with treatment contents (e.g., rape, radical rap) representing particularly compelling clips often shown out of context.

As for the interventions permissible under humansubjects research guidelines, most of the results produced are correlational in nature, leaving open the question of causality. Bearing in mind those limitations in validity and reliability, it's useful to contrast the literatures on rock music content and effects.

\section{INTEGRATING RESEARCH RESULTS}

Across the various contexts reviewed here, as well as other media effects domains, the most commonly used approach to understanding influences on individuals is social learning theory [15]. Based on the work of Bandura [72], the perspective states that mass media present a symbolic field of social information upon which audiences rely to make decisions about their personal lives (e.g., modeling of musician fashion and lifestyles). When outlining the negative effects of heavy metal and rap music, Roberts [19] echoed Bandura's observation concerning the likelihood that youngsters will learn from the media to which they are exposed: "...after the capacity for observational learning has fully developed, one cannot keep people from learning what they have seen" (or heard) [72].

Although widely varying contexts preclude comprehensive meta-analyses of music content and effects, Allen et al. [15] provide the most definitive empirical work on music influences, noting that exposure is weakly related to antisocial actions. The authors caution, however, that only two types of measures could be supported by the minimum of three studies necessary for their meta-analysis. Even in this limited realm where results could be obtained, the authors maintain that such a finding "does not provide complete information necessary for a causal explanation..."

Taken together, this work suggests that the uneven literature on music effects falls to establish a "smoking gun" threshold sufficient to support censorship actions, based on 
the Miller obscenity test or any public nuisance standards [14]. As Christenson and Roberts [16] conclude:

Those who advocate censorship often know well ahead of time that their proposed laws will never withstand judicial review, just as those in the industry who label any government action as unconstitutional know that the Supreme Court has never taken an absolutist position on the First Amendment (p. 227).

The authors' own position seems to fit within the "proregulation" camp [73], which is consistent with Roberts' [19] critical testimony before Senator Brownback's committee on media effects. Even so, this perspective is not inconsistent with Donnerstein et al.'s [74] milder contention that children under 12 are "sexual illiterates" and therefore such material is unlikely to prove harmful; when kids are old enough to have an interest and understanding for such terms as "uterus", they're likely to have developed moral values that mitigate against negative effects. As Christianson and Roberts [16] conclude:

Indeed, even though there may be harm, neither the research we have reviewed...nor the literature on the effects of sexual portrayals in television and movies provides convincing evidence of a major negative influence on moral values or sexual behavior (p. 22).

Yet the authors are less sanguine about the influence of indecent fare than the Donnerstein school. In seeking to "cavil" with the latter's approach, Christenson and Roberts note (1) although a 0- or 10-year-old might not fully understand sexual innuendo, few would have difficulty understanding direct depictions of sex in many popular songs (or Carlin's seven "dirty" words), and (2) effects on language, such as the learning of indecent words, are nevertheless effects.

Drawing from social learning theory, the authors [16] draw several conclusions concerning the extent to which music serves as a dominant, uncontested source of information for an adolescent:

...the likelihood that it will influence his or her beliefs and behavior increases dramatically...When it comes to issues of style, fashion, body image, language, and the nature of friendship--indeed, even suicide and violence-adolescents more often turn to music and MTV than to parents or teachers (p. 223).

Research conducted in the decade since 1998 tends to affirm the pattern of findings uncovered in earlier work. The challenge of establishing these effects empirically (e.g., accreting effects sizes) is greater for research addressing these music modalities than in general studies of television violence, where a much richer literature provides more metaanalytic grist definitional and methodological comparison. Clearly, learning can occur from music, although it operates amidst a complex nexus of influences. The difficult challenge faced by social learning adherents involves the quantum leap from "exposure = learning" to "exposure = antisocial behavior". When drawing their more critical conclusions, maximum effects scholars generally argue beyond what the limited empirical evidence suggests on recorded music and music videos. They must, perforce, rely on general theories of communication (e.g., cultivation, social learning) in order to "fill in the blanks" in the research record.

\section{DISCUSSION}

Perhaps the most compelling treatise on the ills of MTV and of pornography generally is Sut Jhally's (1994) Dreamworlds. Commentators [16] note that this antipornography documentary also illustrates one of the pitfalls underpinning media effects controversies such as this: purposively selected information. In particular, they criticize Jhally's presentation-remade as Dreamworlds 3 in 2007-as a closer approximation to political rhetoric than objective social scientific analysis; such opportunistic presentations of "worst-case" female portrayals of misogyny, etc. represent, according to this view, a one-sided presentation.

These images are implicated, in Jhally's conclusion, as key contributors to an increasingly misogynistic culture in which one in four women are raped in their lives, including one in eight college women (where $84 \%$ of victims know their attacker and $57 \%$ of these happen on dates). Implicit in that analysis is an assumption that the media act as hypodermic needles, injecting audiences with powerful antisocial effects, including rape. This notion receives very little support in the literature [1,17].

In fact, several of the social scientific experiments could be criticized for shortcomings similar to Jhally's. That is, in trying to select a compelling stimulus, the researchers typically rely on "worst case" stimuli (e.g., "Public Enemy" clips featuring violence against women) and present them as "representative" stimuli for a subgenre of music video. The almost exclusive reliance on experimental research designs in the literature, for instance, also presents certain other methodological confounds in the received knowledge on music effects. Given the above propensity to feature poignant, de-contextualized images, researchers are "leading" subjects to express more powerful effects than might be otherwise experienced in a naturalistic (homebased) viewing situation. In fact, the artificiality of the lab setting--where students are often brought into a sterile classroom and told to watch "representative" videos before completing a questionnaire--is quite different from the way audiences consume TV.

Perhaps, for that reason, formal meta-analyses in this realm - to the degree that they could be conducted-confirm the generally modest patterns of effect sizes noted in other media effects domains. In particular, based on Priess et al.'s [17] meta-analysis of variegated realms of media influence, we might not expect to find the proportion of behavior influenced by the media would rarely exceed 5\%. As Allen et al.'s [15] work suggests, the impact of music on behavior is unequivocally antisocial, but this influence is weakened by moderator variables.

Within a given household, for instance, we see "mediating" factors which might ameliorate media effects. These might include intervening co-viewers (especially adults) who either change a channel or provide verbal commentary to help put these images into a proper context 
$[8,36,75]$. Such mediation has been found to attenuate the negative influence of violent portrayals generally, but is absent in artificial lab settings.

Also, lab experiments are notoriously difficult to disguise. Thus, when a set of college students is brought in-typically for (extra) course credit, they may sense that a professor -perhaps one harboring a critical agenda--is seeking to find evidence of antisocial effects. This type of "priming" might, however unwittingly expressed, prompt students to exaggerate self-reported media influences on their behavior.

Another problem with experiments involves the selection of participants. While it could perhaps be argued that college sophomores are also people, experiments obviously present limitations in terms of generalizability of research findings involving these relatively homogenous, captive groups of study participants. To begin, we see a natural skew in age, education, social status and ethnicity (over-representing whites and Asians) that render college students poor approximation of the larger population.

Although one might argue that such "convenience samples" are useful for understanding the peak age groups for music listenership--young adults--a university student is still likely to skew higher than the average citizen on such factors as academic motivation and of course educational attainment. Moreover, studies done on "Gen-X" students are hardly representative of the population as a whole, given that these young adults are likely to rank lower than average in such factors as work motivation.

Owing to the to their enhanced control, work dating to Hovland shows that experiments are more likely to generate stronger relationships than a survey alternative, with pre- and post-test designs allowing for a clearer representation of causality [76]. But these advantages in internal validity obviously come at the cost of the external validity afforded by larger, cross-sectional survey designs. One way to counter the low statistical power associated with some experiments is to conduct a group administration survey with to several hundred children. Although a small number of studies have done this--thus generating more robust, powerful findings-they too are "one-shot" in nature and leave open the issue of causality...an issue of particular importance in music research. Moreover, group administration surveys, as measures of a relatively homogenous population, present questionable vehicles for some of the more powerful statistics (e.g., multiple regression) that could help establish causality. Such analyses typically require more representative data (more closely following general population parameters) that are more readily generalizable.

\section{CONCLUSION}

The present study set out to provide a comprehensive narrative analysis of literature addressing the allied areas of music and music television content as well as effects. Virtually all studies reviewed reveal antisocial themes which are linked, albeit weakly, with negative social outcomes. Acknowledged is the fact that a narrative literature review of this sort cannot provide the kind of "rejection region"characteristic of a formal meta-analysis - that can establish causality (or the lack thereof).
Unfortunately, the variegated contexts in which this research has been conducted mitigates against the accretion of common study measures and study methodologies that can help further the cause of formal meta-analysis [15].

On balance, experiments provide a stronger case for effects [17], but the literature is replete with validity tradeoffs involving laboratory versus survey-based research. Perhaps owing to the limitations in logistics and economic resources, only one of the criterion reviewed studies was conducted with a robust, nationally representative sample that could provide the confidence we need to make generalizations about media effects. However, that study [39] was focused on sexual behavior rather than violence effects.

For these and other reasons, critics of rock music have needed to "fill in the blanks" of their empirical arguments with selective citations to the voluminous literature on general media effects (e.g., with TV violence). Even in that literature--now several thousand studies strong--we see little in the way of longitudinal, externally valid findings that can establish a "smoking gun" with media influences as potent causal agents with human behavior [17].

Krcmar and Stirzhakova's [36] review of work addressing the impact of violence on children, for instance, reports weaker effect sizes than comparable work addressing television. Still, Johnson's [4] analysis suggests that exposure to videogames may prompt other negative outcomes, such as decreases in helping behavior. His work also underscores the importance of considering study context, as methodologically weaker studies yielded smaller effects sizes than stronger studies... a result suggesting that previous meta-analytic studies in this realm may have underestimated the true magnitude of behavioral effects.

Although the stories told in media may potentially play a role on the periphery of a troubled child's life, a strict stimulus-response ("exposure $=$ effect") dynamic fails to explain why Japan, for instance, has a dramatically lower crime rate despite much higher levels of media violence. To be sure, there are several complex socio-cultural variables at play in the determination of crime rates across cultures. But the influence of popular media like rock music on real-life violence has yet to be demonstrated in a sufficiently compelling fashion to warrant government action.

As Jeffres' [10] review of myriad media effects studies suggests, the violence instigation notion is based on a maximum influence dynamic termed the "hypodermic needle" effect [17]. But whether the media causal agent involves TV violence, pornography or public health campaigns, the empirical evidence for a hypodermic needle effect is lacking. In point of fact, recent work in communication and social psychology--much of it beyond the scope of our analysis--suggests that violent crime cannot be easily blamed on the media, poverty or even lax gun laws.

Although human-subjects designs might require a more rigorous standard of measurement/ evidence than rhetorical analysis, they too are capable of leading study results in a similar direction. That is, in trying to select a compelling stimulus, the researchers typically rely on "worst case" stimuli (e.g., "Public Enemy" clips featuring violence against women) and present them as "representative" stimuli for a 
subgenre of music video. That said, new media channels like the internet allow for myriad opportunities for individualized viewing of such contents, and the impact of these emerging inter-mass channels on music consumption should be investigated in later work $[6,36]$.

In sum, just as film was heralded as a potentially destructive new medium during the 1920s [9] emerging media like MTV, videogames and the Internet will continue to attract media scrutiny and attendant social concern [77]. This phenomenon was perhaps most vividly demonstrated by the extensive press coverage of the school shootings in Colorado, and an apparent copy-cat that occurred in Finland in November of 2007.

On the heels of such tragedies, the new media-including emerging online channels through which children now receive music content--will likely emerge as popular targets for regulation $[78,79]$. But the fact that parents can now be charged for the crimes of their children underscores, for now, the degree to which society regards individual and family responsibility to be paramount. It will be important, in later work, to further investigate (a) alternative avenues for scholars to assess the impact of viewing violence on individual and collective psyches, (b) innovative ways in which that impact might be studied, and (c) ways of explaining to authorities that they may be on the wrong track when looking for causes. Further work should address this regulatory dynamic as more new media come online.

\section{ACKNOWLEDGEMENTS}

We would like to thank the anonymous referees for helpful comments and suggestions for this paper.

\section{REFERENCES}

[1] Bryant J, Bryant A. Television and the American family. Mahwah, NJ: LEA 2001

[2] Rosen H. Statement prepared for hearings on the "Social impact of music violence." United States Senate Committee on Governmental Affairs, Subcommittee on Oversight of Government Management, Restructuring, and the District of Columbia: Washington, DC 1999.

[3] Attorney General's Commission on Pornography. Washington D.C.: Government Printing Office 1986.

[4] Johnson S. Everything bad is good for you. New York: Riverhead Books 2005.

[5] Striesand B. Lawyers guns, money. U.S. News and World Report 1999: 56-7.

[6] Lin C, Atkin D. Communication technology and social change. Mahwah, NJ: LEA 2007.

[7] Abelman R, Lin CA, Atkin D. A meta-analysis of television's impact on special populations. In: Preiss R, Gayle B, Burrell N, Allen M, Bryant J, Eds. Mass media theories and processes: Advances through meta-analysis. Mahwah, NJ: LEA 2007, pp. 111127.

[8] Atkin D, Greenberg BS, Baldwin TF. The home ecology of television viewing: Parental mediation and the new media environment. J Commun 1991; 41: 40-53.

[9] Wartella E, Reeves B. Historical trends in research on children and the media: 1900-1960. J Commun 1985; 35: 118-35.

[10] Jeffres L. Mass media effects. Prospect Heights, IL: Waveland 1997.

[11] Lieberman J. Statement prepared for Senate hearings on "the social impact of music violence". Senate subcommittee on oversight of government management, restructuring, and the District of Columbia: Washington, DC, Nov. 6, 1997.

[12] Telecommunications Act of 1996. 47 U.S.C. 1996.

[13] Reno v. the American Civil Liberties Union. 25 Med. L. Rptr. 1833, 1997.
[14] Van Alstyne W. The American first amendment in the twenty-first century. New York: Foundation Press, 2002.

[15] Allen M, Jorgenson J, Ryan DJ, Herrett-Skjellum J, Kramer MR, Timmerman L. Effects of music. Mass media effects research: Advances through metaanalysis. Mahwah, NJ: LEA 2007.

[16] Christenson PG, Roberts, D. It's not only rock and roll: Popular music in the lives of adolescents. Cresskill, NJ: Hampton 1998.

[17] Priess R, Gayle B, Burrell N, Allen M, Bryant J Eds. Mass media theories and processes: Advances through meta-analysis. Mahwah: NJ: LEA 2007.

[18] Dixon T, Linz D. Obscenity law and sexually explicit rap music: Understanding the effect of sex, attitudes, and beliefs. J Appl Commun Res 1997; 25: 217-41.

[19] Roberts DF. Research of adolescent uses of and responses to heavy metal and rap music. Testimony prepared for Senate Hearings on "The Social impact of music violence". Washington, D.C. Nov. 6, 1997.

[20] Rice R. The content of popular recordings. Popular Music Soc 1980; 7(2): 140-58

[21] Hyden C, McCandless N. (1983). Men and women as portrayed in popular music lyrics. Popular Music Soc 1983; 9 (2): 19-26.

[22] Albert WG. Dimensionality of perceived violence in rock music: Musical intensity and lyrical violence content. Popular Music Soc 1978; 6: 27-38.

[23] Roberts DF, Kinsey D, Gosh S. Themes in top 40 songs of the 1980's. Unpublished raw data 1993.

[24] Vincent RC, Davis D, Boruszkowski, LA. Sexism on MTV: The portrayal of women in rock videos. Journal Q 1987; 64: 750-5.

[25] Jhally S. Dreamworlds II: Desire, sex and power in music video. Northampton, MA: Media Education Foundation (video) 1995.

[26] Kalis, P, Neuendorf K. Aggressive cue prominence and gender participation in MTV. Journal Q 1989; 66: 148-54, 229.

[27] National Coalition on Television Violence. NCTV Music-video monitoring project (Research report). Marlboro, MA: Author 1984.

[28] Sherman B, Dominick JR. Violence and sex in music videos: TV and rock 'n' roll. J Commun 1986; 63: 79-84.

[29] Baxter RL, De Riemer C, Landini A, Leslie L, Singletary L. A content analysis of music videos. J Broadcast Electron Media 1985; 29: 333-40.

[30] Davis D. Nihilism in music television. Paper presented at the annual meeting of the Speech Communication Association, Chicago, IL 1984

[31] Brown J, Campbell K. Race and gender in music videos: The same beat but a different drummer. J Commun 1986; 36: 94-106.

[32] Macdonald PT, Estep RE. Criminal violence on prime-time TV and MTV. Paper presented at the Society for the Study of Social Problems 1989.

[33] Tapper J, Thorson E, Black D. Variations in music videos as a function of their musical genre. J Broadcast Electron Media 1994; 38: 103-13.

[34] Jones K. Are rap videos more violent? Style differences and the prevalence of sex and violence in the age of MTV. Howard J Commun 1997; 8 (4): 343-56.

[35] Fiore F. Prevalence of TV film violence is studied. Los Angeles Times, Sep 23, 1999.

[36] Krcmar M, Stirzhakora Y. Computer-mediated technology and children. In: Lin CA Atkin D, Eds. Communication technology and social change. Mahwah, NJ: LEA 2007, pp. 57-76.

[37] Roberts DF, Foehr UG, Rideout V. Generation M: Media in the lives of 8-18 year-olds. Menlo Park, CA: Henry J Kaiser Foundation 2003. Available at: www.kff.org/entmedia/ entmedia030905pkg.cfm. Accessed February 15, 2008.

[38] Peterson SH, Wingood GM, DiClemente RJ, Harrington K, Davies S. Images of sexual stereotypes in rap videos and the health of African American female adolescents. J Women's Health 2007; 16: 1157-77.

[39] Martino SC, Collins RL, Elliott MN, Strachman MA, Kanouse DE, Berry SH. Exposure to degrading versus nondegrading music lyrics and sexual behavior among youth. Pediatrics 2006; 118: 430-41.

[40] Burke R, Grinder R. Personality-oriented themes and listening patterns in teen-age music and their relation to certain academic and peer variables. School Rev 1966; 74: 196-211.

[41] Lewis G. Popular music, musical preference and drug use among youth. Popular Music Soc 1980; 7: 176-81.

[42] Larson R, Kubey R. Television and music: Contrasting media in adolescent life. Youth Soc 1983; 15: 13-31. 
[43] Tanner J. Pop music and peer groups: A study of Canadian high school student's responses to pop music. Can Rev Sociol Anthropol 1981; 18: 1-13.

[44] Prinsky LE, Rosenbaum JL. Leer-ics or lyrics: Teenage impressions of rock 'n' roll. Youth Soc 1987: 18: 384-97.

[45] Wanamaker CE, Reznikoff M. Effects of aggressive and nonaggressive rock songs on projective and structured tests. J Psychol 1989; 123: 561-70.

[46] St. Lawrence JS, Joyner DJ. The effects of sexually violent rock music on males' acceptance of violence against women. J Psychol Women Q 1991; 15: 49-63.

[47] Arnett J. Adolescents and heavy metal music: From the mouths of meatheads. Youth Soc 1991; 23: 76-83.

[48] Arnett J. Heavy metal music and reckless behavior among adolescents. Youth Adolescence 1991; 20: 573-92.

[49] Bleich S, Zillman D, Weaver J. Enjoyment and consumption of defiant rock music as a function of adolescent rebelliousness. Broadcast Electron Media 1991; 35: 351-66.

[50] Johnson JD, Adams MS, Ashburn L, Reed W. Differential gender effects of exposure to rap music on African American adolescents' acceptance of teen dating violence. Sex Roles 1995; 33 (7-8): 597605.

[51] Smith B. The effects of exposure to violent lyric music and consumption of alcohol on aggressiveness. Diss Abstr Int B Sci Eng 1995; 56 (6-B): 3487.

[52] Barongan C, Hall G, Nagayama C. The influence of misogynous rap music on sexual aggression against women. Psychol Women Q 1995; 19 (2): 195-207.

[53] Webster SR, Crown CL, Quatman GL, Heesacker, M. The influence of sexually violent rap music on attitudes of men with little prior exposure. Psychol Women Q 1997; 21: 497-508.

[54] Trostle L. Nihilistic adolescents, heavy metal rock music and paranormal beliefs. Psychol Rep 1986; 59: 10.

[55] Martin G, Clarke M, Pearce C. Adolescent suicide: Music preference as an indicator of vulnerability. Acad Child Adolescent Psychiatry; 32 (3): 530-35.

[56] Stack S, Gundlach J, Reeves K. The heavy metal subculture and suicide. Suicide Life Threat Behav 1994; 24: 15-24

[57] Stack S, Gundlach J. The effect of country music on suicide. Social Forces 1992; 71: 211-8.

[58] Ballard ME, Coates S. The immediate effects of homicidal, suicidal, and nonviolent heavy metal and rap songs on the moods of college students. Youth Soc 1995; 27 (2): 148-68.

[59] Brown JD, L'Engle KL, Pardun CJ, Guo G, Kenneavy K, Jackson C. Sexy media matter: exposure to sexual content in music, movies, television, and magazines predicts black and white adolescents' sexual behavior. Pediatrics 2006; 117: 1018-27.

[60] Sun S, Lull J. The adolescent audience for music videos and why they watch. J Commun 1986; 36: 115-25.
[61] Greeson LE, Williams RA. Social implications of music videos for youth: An analysis of content and effects of MTV. Youth Soc 1986; 18: $177-89$.

[62] Walker JR. How viewing of MTV relates to exposure to other media violence. Journal Q 1987; 64: 756-62.

[63] Peterson DL, Pfost KS. Influence of rock videos on attitudes of violence against women. Psychol Rep 1989; 64: 319-22.

[64] Hanson CH, Hanson RD. Rock music videos and antisocial behavior. Basic Appl Psychol 1990; 11: 357-69.

[65] Hanson CH, Hanson RD. The influence of sex and violence on the appeal of rock music videos. Commun Res 1990; 17: 212-34.

[66] Miller MA. The effects of music videos on adolescent meaning construction and attitudes toward physical violence as a method of conflict resolution. ERIC number ED330955 1990.

[67] Waite B, Hillbrand M, Foster H. Reduction of aggressive behavior after removal of music television. Hosp Commun Psychiatr 1992; 43:173-5.

[68] Wallbott HG. Sex, violence, and rock'n'roll--zur rezeption von musikvideos unterschiedlichen inhalts. (...On the reception of videoclips with different contents). Medienpsychologies: Zeitschrift fuer individual- and massenkommunikation 1992; 4(1): 3-14.

[69] Zillman D, Aust CF, Hoffman KD, et al. Radican rap: Does it further ethnic division? Basic Appl Soc Psychol 1995; 16: 1-25.

[70] Johnson JD, Jackson LA, Gatto L. Violent attitudes and deferred academic aspirations: Deleterious effects of exposure to rap music. Basic Appl Soc Psychol 1995; 16 (1-2): 27-41.

[71] Abelman R. Can we generalize from generation X? Not! Broadcast Electron Media 1996; 40: 441-6.

[72] Bandura A. Social learning theory. Englewood Cliffs, NJ: PrenticeHall 1973.

[73] Roberts DF, Christenson, PG. Popular music in childhood and adolescence. In: Singer DG, Singer JL, Eds, Handbook of children and the media. Thousand Oaks, CA: Sage Publications, Inc, pp. 395-414.

[74] Donnerstain E, Linz D, Penrod S. The question of pornography: Research findings and policy implications. New York: Free Press 1987.

[75] Abelman R, Pettey G. Child attributes as determinants of parental television-viewing mediation. Family Issues 1989; 10: 251-66.

[76] Stempel GH, Weaver DH, Wilhoit GC. Mass communication research and theory. Boston: Allyn and Bacon 2003.

[77] Anderson CA. An update on the effects of playing violent videogames. Adolescence 2004; 27: 113-22.

[78] McLeod DM, Eveland WP Jr, Nathanson AI. Support for censorship of violent and misogynic rap lyrics: An analysis of the third-person effect. Commun Res 1997; 24: 153-74.

[79] Struasberg VC, Wilson B. Children, adolescents and the media. Beverly Hills: Sage Publications, Inc 2002.

This is an open access article licensed under the terms of the Creative Commons Attribution Non-Commercial License (http: //creativecommons.org/licenses/by$\mathrm{nc} / 3.0 /$ ), which permits unrestricted, non-commercial use, distribution and reproduction in any medium, provided the work is properly cited. 